\title{
Un análisis de la reacción me gusta en Facebook desde los estudios de la interacción
}

An analysis of the Facebook like reaction from interaction studies

Asela Reig Alamillo

Universidad Autónoma del Estado de Morelos, Centro de Investigaciones en Ciencias Cognitivas

Alejandro Elizondo Romero Universidad Autónoma Metropolitana-Iztapalapa, Universidad Veracruzana

\section{Resumen}

El artículo analiza el empleo del emotico- de principios pragmáticos, en concreto, los no reactivo me gusta en la red social Fa- conceptos de pertinencia condicional, rescebook a partir de conceptos teóricos de puesta preferida o no preferida, la búsquela pragmática y el análisis de la conversación. Se describe el empleo de me gusta como una intervención reactiva cuya interpretación (literal, como valoración positiva de un contenido, o no literal) depende da de relevancia y las características de los participantes y su relación dentro de la red social, los cuales permiten explicar el empleo y la interpretación literal o no literal del emoticono en interacción. de las características de la interacción y

Palabras clave: comunicación mediada por computadora (CMC); interacción en redes sociales; emoticonos; reacciones en Facebook 


\section{Abstract}

This article analyzes the use of the like concepts of conditional relevance, prereaction button in Facebook by apply- ferred/non-preferred answer, pursuit of ing theoretical concepts from Pragma- relevance, and the profile of participants tics and Conversation Analysis. The use and their relationships within the soof like is described as a responsive act cial networking, all of which explain the whose interpretation (either literally, as a use and the literal/non-literal interpretapositive assessment, or non-literally) de- tion of the like reaction button in Facepends on both features of the interaction book interactions. and pragmatic principles, specifically, the

Keywords: computer-mediated communication (CMC); social networking interaction; emoticons; reaction buttons in Facebook 


\section{Introducción}

La comunicación a través de dispositivos electrónicos ha pasado a ocupar un papel crucial en la sociedad y, como resultado, ha surgido en los últimos años la disciplina dedicada a la comunicación mediada por computadora (CMC), es decir, la comunicación producida cuando los humanos interactúan entre sí transmitiendo mensajes a través de computadoras conectadas a una red. El discurso mediado por computadora es una subdisciplina de la CMC que se centra en el estudio del lenguaje y su uso en redes computacionales, empleando los métodos del análisis del discurso (Herring, 2001: 612). Esta disciplina analiza las particularidades de las nuevas formas de comunicación a través del correo electrónico, páginas web, redes sociales, chats, blogs, etcétera, y, para ello, aplica los conocimientos generados desde la pragmática y el análisis del discurso. Este es el marco del presente trabajo, en el que se aplican conceptos desarrollados en pragmática y, concretamente, en el análisis de las interacciones y el análisis conversacional, a un fenómeno específico de la comunicación realizada a través de la red social Facebook: el empleo del emoticono reactivo me gusta.

El empleo de dicho emoticono en Facebook y la reciente ampliación del repertorio de emoticonos han despertado curiosidad entre analistas de la computación y comunicólogos, quienes observan las tendencias de uso de estas y otras herramientas de la red social. Sin embargo, este fenómeno no se ha abordado poniendo en juego los conocimientos que desde la lingüística se tienen respecto a las interacciones y sus componentes, así como a la interpretación que los hablantes otorgan a las intervenciones en un intercambio comunicativo según la posición que ocupen en la interacción.

Este trabajo analiza el empleo del emoticono reactivo me gusta como un componente de los intercambios comunicativos que se llevan a cabo a través de Facebook. El análisis pretende contribuir desde la lingüística a la descripción y estudio de la CMC y, a su vez, contrastar la teoría que emerge principalmente del estudio de interacciones cara a cara a interacciones con otras características (comunicación no oral o no exclusivamente oral; emisor y destinatario que no comparten tiempo y espacio, etc.) en las que, además de intervenciones lingüísticas, son frecuentes otro tipo de intervenciones, como las realizadas por medio de imágenes, videos o símbolos no lingüísticos (por ejemplo, los emoticonos). 
Esta perspectiva aplicada permitirá explicar las diferentes interpretaciones que el emoticono reactivo me gusta recibe y cómo son condicionadas por factores concernientes a la pragmática y al análisis discursivo — búsqueda de relevancia y asunción del papel cooperativo y no conflictivo del interlocutor-, además del contenido semántico convencionalmente asignado al emoticono.

El resto del artículo está organizado de la siguiente manera. En §2 se presenta una breve introducción de la red social Facebook y de las reacciones mediante emoticonos. En $\S 3$ se revisan algunos conceptos teóricos básicos sobre la interacción y sus componentes. Posteriormente, se caracterizan los emoticonos de Facebook como una intervención reactiva ( $\$ 4)$ y se aborda en $\$ 5$ el análisis de la interpretación del me gusta; en §5.1. se analizan las condiciones de adecuación de su empleo con significado literal y en $\$ 5.2$. se abordan las interpretaciones no literales. Por último, en $\S 6$, el artículo presenta las consideraciones finales sobre los nuevos emoticonos introducidos en la red social y las conclusiones del estudio.

\section{Facebook}

La red social Facebook fue fundada el 4 de febrero de 2004 por Mark Elliot Zuckerberg, y se ha convertido en la red con el mayor número de usuarios en el mundo, aproximadamente 2200 millones de personas activas mensualmente. ${ }^{1} \mathrm{La}$ red permite interactuar a través de mensajes publicados en la página principal de cada usuario, los cuales pueden ser leídos por otros usuarios previamente autorizados como miembros de su círculo social.

Según el modelo de clasificación propuesto por Herring (2007), el modelo de facetas, a continuación podemos ofrecer una caracterización más concreta de Facebook. Desde el punto de vista técnico, se trata de una red principalmente asincrónica en donde los mensajes son enviados de forma unidireccional, es decir, que el mensaje llega como un bloque completo solo cuando el emisor ha finalizado del todo su construcción. La longitud de los mensajes enviados puede ser considerablemente larga, alrededor de 63206 caracteres al día de hoy, aunque con cada actualización de la red el número suele incrementarse. Dichas

1 Cifra actualizada a marzo de 2018, tomada del sitio de Facebook: https://newsroom.fb.com/ company-info/ 
emisiones no cuentan con una persistencia predeterminada por el sistema, lo que hace posible revisitar mensajes después de años.

La naturaleza de las emisiones es completamente multimodal al integrar una gran cantidad de canales. Las intervenciones reactivas pueden incluir texto, imágenes, hipertexto o videos, pero el usuario puede también optar por responder únicamente con lo que se ha denominado me gusta.

En cuanto a los participantes, el sistema permite la filtración y clasificación de los receptores en cada mensaje o de manera general agrupándolos en amigos, conocidos o restringidos.

El último de los rasgos técnicos propuestos por Herring (2007) es el que se refiere a la posibilidad de citar mensajes ajenos o propios dentro de un nuevo mensaje acompañado, o no, de un nuevo comentario por parte del emisor más reciente.

En cuanto a los factores situacionales, es posible determinar que las interacciones generalmente se llevan a cabo en un modelo de uno a muchos, es decir, un solo emisor con múltiples receptores posibles muy poco delimitados.

\subsection{Las reacciones mediante emoticonos en Facebook}

Una de las características distintivas de esta red social es el emoticono que se ha denominado me gusta (Figura 1), cuya imagen (mano cerrada con pulgar arriba) ha llegado a ser una marca característica de la red. Dicho icono permite al receptor reaccionar positivamente a un mensaje determinado, sea cual sea la naturaleza de este (imagen, vínculo, video, etc.), simplemente indicando por medio del emoticono reactivo me gusta que dicho mensaje le gusta o agrada.

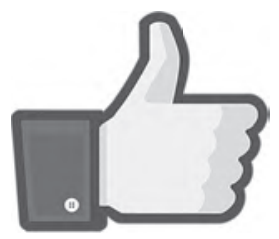

Figura 1. Emoticono reactivo de me gusta en Facebook

En la red surgieron publicaciones en las que los usuarios solicitaban a Facebook que ofreciera un emoticono no me gusta, para poder responder a intervenciones 
con la reacción contraria (Figura 2). En su lugar, el 24 de febrero de 2016, Facebook liberó al mercado global cinco botones adicionales al me gusta con el fin de ampliar las posibilidades de expresión de los usuarios (Figura 3). Los nuevos emoticonos satisfacían la necesidad de tener una reacción frente a publicaciones con contenidos negativos y ofrecían, además, otras reacciones positivas.

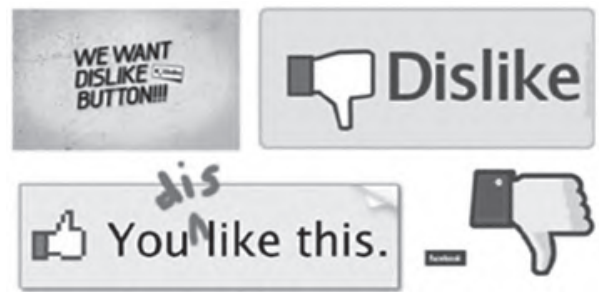

FIgURA 2. Ejemplos de peticiones del emoticono no me gusta en internet

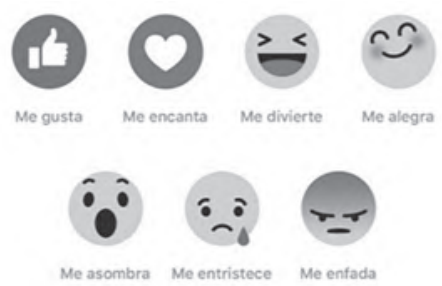

Figura 3. Nuevos emoticonos reactivos de Facebook

Algunos análisis cuantitativos realizados tiempo después de implementar estos cambios $^{2}$ mostraron que las reacciones por medio de emoticonos constituían $78.4 \%$ de las intervenciones reactivas en los datos analizados. El restante $21.6 \%$ se conforma de comentarios (7.2\%) y de "compartir" las publicaciones (14\%). Dentro de las reacciones por medio de emoticonos, en los datos analizados en mayo de 2016 solo $2.4 \%$ correspondían a emoticonos que no fueran me gusta y, pocos meses después, en junio del mismo año, este porcentaje se elevó a 7.1\%.

2 Datos obtenidos de Quintly. Estudio realizado de mayo a septiembre de 2016, a partir del análisis de 130000 posts. Véase https://www.quintly.com/blog/2016/05/Facebook-reactions-study/ 
La frecuencia de las intervenciones por medio de emoticonos es, por tanto, muy alta en el conjunto de las interacciones realizadas en Facebook y, en concreto, meses después del surgimiento de los nuevos emoticonos, el me gusta sigue siendo el más usado con mucha diferencia. En los siguientes apartados veremos que el frecuente empleo de me gusta se explica por la capacidad que los usuarios tienen para asignar una interpretación no literal al empleo de este emoticono. Dar cuenta de las posibles interpretaciones del me gusta en la interacción en Facebook y de los mecanismos que permiten llegar a ellas requiere que revisemos algunos conceptos básicos del funcionamiento de las interacciones.

\section{Interacción y movimientos}

La noción de interacción, importada al campo de la lingüística desde la sociología, designa en primer lugar un proceso de intercambio de acciones y reacciones, aunque se utiliza normalmente para designar, por metonimia, a cierto tipo de objeto caracterizado por la presencia masiva de este proceso (Kerbrat, 1998: 54-55).

El amplio concepto de interacción incluye intercambios tan variados como ir al mercado, circular en coche, pedir información telefónica, jugar al futbol, realizar un trámite burocrático o acordar una cita con un amigo. Se trata de situaciones muy diferentes, pero durante todas ellas es necesario interactuar, esto es, actuar conjuntamente con otra u otras personas. En algunas de estas situaciones nos servimos de la lengua como modo principal de relacionarnos con el otro participante mientras que, en otras, la interacción es producto de un intercambio de acciones no verbales. Se distingue, así, entre interacciones verbales (aquellas que se realizan principalmente por medios lingüísticos) e interacciones no verbales (en las que el intercambio se realiza por medios no lingüísticos). Es frecuente, sin embargo, que en la interacción coexistan acciones verbales y no verbales, produciendo lo que Kerbrat llama una interacción mixta (1990: 135; 1996: 7). En estos casos, ambas acciones se mezclan y son igualmente necesarias para el desarrollo de la interacción. Por ejemplo, en una panadería, la interacción que tiene lugar entre el cliente y el panadero es una interacción mixta, en la que coexisten acciones verbales (el cliente pide el pan que 
desea comprar o el panadero dice el precio) y acciones no verbales (el panadero da el pan al cliente, el cliente paga o el panadero le da el cambio).

En el contexto de la CMC, el canal de comunicación preferido por los usuarios es la lengua escrita, a pesar de que es posible interactuar también de manera oral. En este tipo de comunicación, las interacciones mixtas son frecuentes y se integran principalmente por intervenciones lingüísticas, tanto orales como escritas, e intervenciones gráficas con imágenes de distintas características.

Hasta aquí hemos hablado de las acciones que los participantes realizan en la interacción, acciones mediante las cuales se relacionan los participantes. Desde la sociología, y después en el análisis de la conversación, se describe que los participantes van tomando el turno en la interacción (concebido como un hueco estructural) y lo van llenando con intervenciones. La intervención es, por tanto, la contribución de un hablante a una interacción al llenar un turno y está limitada por dos cambios de hablante (Gallardo, 1996: 46). A su vez, cada intervención está formada por uno o más movimientos.

El término movimiento es introducido por el sociólogo Erving Goffman (1955) para nombrar a la unidad mínima de naturaleza interactiva. Este término no se refiere exclusivamente a actividades lingüísticas sino también a actividades físicas, aunque se ha utilizado mucho para el análisis de las interacciones verbales y en el análisis de la conversación se utiliza para referirse a acciones verbales. Así, Gallardo (1996: 79) lo define como "el acto o conjunto de actos de habla dotados de valor interactivo, es decir, que involucran a un hablante y un oyente" y añade que "son las unidades en las que puede fragmentarse una intervención". ${ }^{3}$ Cualquier interacción puede descomponerse en movimientos, acciones verbales o no verbales de los participantes que hacen avanzar la interacción. Por ejemplo, en una situación en la panadería podríamos describir la interacción como:

3 Gallardo (1996: 79) señala aquí un problema terminológico: en inglés se utiliza el término move para lo que en castellano se conoce como intervención, y nosotros hablamos de movimiento para indicar lo que Coulthard (1977) llama act: un turno o fragmento de turno que realiza una determinada función en relación con los turnos del entorno inmediato. Más recientemente, en español también se usa movimiento como distinto a intervención en la gramática discursivo-funcional y otros enfoques del discurso (Hengeveld \& Mackenzie, 2008; Alturo, 2010). 
Intervención 1: Movimiento 1: el cliente saluda Movimiento 2: el cliente pide dos barras de pan

Intervención 2: Movimiento 3: el dependiente le entrega las barras Movimiento 4: el dependiente dice el precio de las dos barras

Intervención 3: Movimiento 5: el cliente da el dinero

Intervención 4: Movimiento 6: el dependiente da el cambio Movimiento 7: el dependiente da las gracias

Intervención 5: Movimiento 8: el cliente coge el cambio Movimiento 9: el cliente se marcha

Los intercambios comunicativos pueden definirse como aquellas formas de interacción cuyo medio para provocar la reacción del interlocutor se constituye por un código conocido por ambos participantes. El principal de estos códigos es el lingüístico, de modo que podemos afirmar que las interacciones verbales son los intercambios comunicativos más frecuentes.

En la comunicación que se lleva a cabo en redes sociales, el código no verbal más usado es el de los denominados emoticonos. Se trata de secuencias de caracteres como signos de puntuación o signos matemáticos que son interpretados como imágenes y no como código lingüístico, de forma que es posible interpretar la secuencia : no como dos puntos y un paréntesis, sino como una cara triste.

De acuerdo con Novak, Smailović, Sluban y Mozetič (2015), los emoticonos se pueden encontrar desde el siglo XIX en la escritura informal y principalmente con fines humorísticos. En escritura mediada por computadora se atribuye su implementación al profesor Scott Fahlman, quien el 19 de septiembre de 1982, en un mensaje publicado en un foro de discusión, propuso marcar los mensajes con la secuencia : - ) o : - ( para delimitar si debían leerse en tono de broma o con seriedad.

Actualmente, las secuencias de caracteres conviven con lo que se conoce como emoji (palabra de origen japonés que literalmente significa 'imagen letra') y su uso se ha popularizado especialmente en la comunicación a través de dispositivos móviles y en servicios de redes sociales. 


\subsection{Intervenciones iniciativas y reactivas, la pertinencia condicional y el concepto de preferencia}

El contenido de las intervenciones y de los movimientos que las componen puede analizarse en términos de actos y, si se realizan lingüísticamente, en términos de actos de habla.

Probablemente, el aspecto más interesante al observar las intervenciones - y sus movimientos compositivos- en la interacción es atender la relación que una intervención mantiene con las demás durante el mismo intercambio y, en concreto, el hecho de que determinado tipo de movimiento espera determinado tipo de respuesta. Estas intervenciones, que intuitivamente se espera que ocurran de manera conjunta, constituyen los llamados pares adyacentes, que Sacks, Schegloff y Jefferson (1974: 716-717) consideraron la unidad mínima de la conversación. El par adyacente se define como un intercambio formado por dos intervenciones, iniciativa y reactiva, producidas en turnos contiguos por distintos hablantes, de manera que un tipo concreto de primera parte del par (intervención iniciativa) requiere un tipo concreto de segunda parte (intervención reactiva). El ideal de este intercambio prototípico sería que la primera y la segunda parte del par se produjesen una tras otra, pero en la práctica no es necesario que se dé dicha adyacencia o contigüidad, pues la relación que une las dos partes es realmente una relación de dependencia que se ha llamado "pertinencia condicional" (conditional relevance). ${ }^{4}$ Esta relación explica que un enunciado en el que se realiza una pregunta condicione la aparición de una segunda parte del par adyacente en el que se dé la respuesta. Schegloff explica la pertinencia condicional de la siguiente manera:

By conditional relevance of one item to another we mean: given the first, the second is expectable; upon its occurrence it can be seen to be a second item to the first; upon its nonoccurrence it can be seen to be officially absent - all this provided by the occurrence of the first item (Schegloff, 1968: 364). ${ }^{5}$

4 Gallardo llama a la relación de dependencia entre dos enunciados “orientación interactiva” (Gallardo, 1998: 19).

5 "Por pertinencia condicional de un elemento respecto a otro queremos decir: dado el primero, 
La pertinencia condicional, por tanto, se refiere a la relación de dependencia que existe entre los actos que se realizan en las intervenciones: entre una pregunta y su respuesta o entre un ofrecimiento y la aceptación o rechazo; en este sentido, se define generalmente en términos de "expectativa" de una respuesta. A esta expectativa, no obstante, se le reconoce una fuerza casi deóntica o, al menos, contractual, pues es más bien el resultado de una suerte de contrato conversacional que, o se sigue, o se rompe notoriamente. En este sentido, Stivers (2013: 192), al definir los pares adyacentes, señala que "con acciones particulares, los actores sociales imponen una obligación normativa en los co-interactuantes de realizar una respuesta de determinado tipo en la primera oportunidad posible" (énfasis añadido). Para referirnos a esta relación de pertinencia condicional en las interacciones cara a cara, especialmente dialógicas, hablaremos por tanto de que un tipo de intervención en el turno iniciativo requiere, en el sentido recién explicado, un tipo determinado de intervención en el turno reactivo. Como veremos en $\S 4$, consideramos que el grado en que se espera o requiere el turno reactivo es diferente según el tipo de interacción.

En virtud del o de los movimientos que las componen, existen intervenciones iniciativas, intervenciones reactivas o intervenciones reactivo-iniciativas. Lo crucial en este concepto es que las intervenciones iniciativas no están predichas por (y no reaccionan a) una intervención anterior, mientras que las intervenciones reactivas son necesariamente no iniciales y sí están predichas, es decir, su aparición está condicionada por una intervención anterior.

No todas las posibles respuestas, pertinentemente condicionadas por una intervención iniciativa, son equivalentes. Una vez asumido que una pregunta requiere la aparición de un acto reactivo que dé la respuesta a esta pregunta, nos encontramos con dos posibilidades, como puede observarse en (1).
(1) A: ¿Me acompañas a comprar?
B: Sí, claro / Lo siento, es que no puedo

el segundo es esperable; dada su aparición, puede verse como un segundo elemento respecto al primero; dada su no aparición, puede verse como oficialmente ausente — todo esto, por la ocurrencia del primer elemento" (la traducción es nuestra). 
Para dar cuenta de este hecho, Sacks (1992) introduce el concepto de preferencia (preference): para cada acto iniciativo existe una posible respuesta preferida y otra no preferida. Sacks se interesa por cómo, según se diseñen las primeras partes del intercambio, las respuestas preferidas y no preferidas de este acto iniciativo serán unas u otras. Así, si diseñamos una pregunta con la forma: Vienes mañana, ¿verdad? hacemos que la respuesta preferida sea sí, mientras que si creamos la pregunta con la forma No vienes mañana, ¿verdad? la respuesta preferida es no. Existe en la interacción, según el autor, una preferencia por la conformidad y una preferencia por la contigüidad: se prefiere un turno reactivo que muestre conformidad y, si este se da, se prefiere que se dé inmediatamente después del turno iniciativo — de forma contigua-, mientras que si se opta por la no conformidad en el turno reactivo, esta tiende a alejarse del turno iniciativo.

Los analistas de la conversación insisten en señalar que el concepto de preferencia se refiere a un concepto técnico, un principio conversacional abstracto, y no a un estado o predisposición psicológica de los participantes de la interacción. En tanto que concepto técnico, se afirma que la preferencia se identifica principalmente por la marcación formal: normalmente, la respuesta preferida se da explícitamente, de forma inmediata y sin rodeos, como en B1 del ejemplo (2), mientras que la no preferida se construye en general indirectamente, se suele retrasar, se presenta normalmente alguna justificación y es frecuente que se utilicen actos de habla indirectos, como en B2.

(2) A: ¿te vienes a la playa?

B1: Ay / sí / qué bien

B2: Ay, es queee -no sé / no me apetece mucho, la verdad. (Gallardo, 1996: 110)

Estos dos rasgos de la preferencia (marcación lingüística y disociación del estado o predisposición psicológica del hablante) son compartidos por muchos autores (Atkinson \& Heritage, 1984; Heritage, 1984; Goodwin \& Heritage, 1990; Hutchby \& Wooffitt, 1998).

Sin embargo, Church (2004) hace patente el hecho de que resulta difícil, incluso para los estudios clásicos sobre preferencia, mantener tan claramente separadas en la práctica la preferencia psicológica de los usuarios y el concepto abstracto relativo a la organización secuencial de preferencia y que, a pesar 
de los esfuerzos que se han hecho por mantener separados los dos conceptos de preferencia —esfuerzos quizá excesivos, en sus palabras-, no resulta claro que estos dos sean necesariamente independientes (Church, 2004: 118). En su opinión, el hecho de que los deseos de los participantes y la forma lingüística marcada se solapen muy frecuentemente provoca problemas para mantener la definición más conservadora que niega inferencias psicológicas.

Church (2004) también afirma que la idea de que existe una especie de principio abstracto de preferencia que opera en las conversaciones es compatible con la idea de que este concepto de preferencia se construye sobre convenciones subyacentes respecto a expectativas sociales más amplias, compartidas por los interactuantes que, como veremos en \$5.2.2., se han entendido en trabajos previos como el deseo de preservar la imagen del interlocutor.

La preferencia, por otra parte, debe juzgarse o calcularse en el contexto local en el que tiene lugar la intervención. Esto quiere decir que no puede asignarse, por ejemplo, a una valoración positiva el rasgo de "acto preferido" en sí mismo, sino que su calidad de "preferido" o "no preferido" dependerá de a qué tipo de turno iniciativo sigue en la interacción (Pomerantz, 1984). Los trabajos previos, por tanto, rechazan en general asignar la característica de preferido o no preferido a tipos de turnos ( $c f r$. Pillet-Shore, 2017), pero sí describen la relación entre actos reactivos y actos iniciativos, y cómo para determinados tipos de acto iniciativo, cierto acto reactivo supone una respuesta preferida o no preferida. Se caracterizan como respuestas preferidas, por ejemplo, dar la información que se pide en una pregunta, obedecer una orden, realizar lo que se solicita en una petición, aceptar una invitación o mostrar acuerdo con una información y son no preferidas negar la información que se pregunta, no obedecer a una orden, no realizar lo que se pide en una petición, declinar una invitación o mostrar desacuerdo con una información.

\section{Caracterización de los emoticonos en Facebook como intervenciones reactivas}

Partiendo del rol que cumplen en la interacción que los usuarios mantienen en la red social Facebook, los emoticonos reactivos asociados con me gusta constitu- 
yen herramientas con las que los usuarios emiten una intervención no lingüística que tiene los siguientes rasgos.

En primer lugar, es necesariamente reactiva, es decir, se emite como respuesta a una intervención anterior, sea esta lingüística o no lingüística y se haya producido en el perfil del usuario o en cualquier otra posición dentro de los comentarios. Asimismo, no puede responderse a estas intervenciones, de forma que además de ser reactiva tiene el rasgo [+final], lo que la diferencia de otras intervenciones reactivas en la misma red social: los comentarios y los actos de "compartir".

En segundo lugar, el emoticono supone un movimiento y no puede combinarse en una misma intervención con otros emoticonos, aunque el mismo usuario podría, además, comentar o compartir.

En tercer lugar, respecto a su contenido, estas respuestas tienen un significado convencional que se recogió en la Figura 3. En términos de una propuesta de actos de habla, entonces, atendiendo al contenido explícito del mensaje diríamos que los usuarios, al emplear, por ejemplo, el emoticono asociado al significado 'triste', realizan un acto de aseverar que está triste o, con más precisión, de aseverar que el contenido al que se reacciona causa tristeza.

El cuarto elemento que caracteriza a estas intervenciones, más interesante desde el punto de vista de la idiosincrasia de la comunicación mediada por computadora, tiene que ver con la pertinencia condicional y, en este sentido, la necesidad de que estos turnos reactivos aparezcan o, dicho de otro modo, las consecuencias de que no aparezcan. Como se describió antes, en la conversación y otras interacciones orales la segunda parte de los pares adyacentes es requerida, de forma que su ausencia es significativa y, como tal, recibe una interpretación. $\mathrm{Si}$, en una conversación como en (3), un hablante realiza un juicio de valor y su interlocutor no ofrece respuesta, el silencio se interpreta, en este caso, como una respuesta no preferida — una falta de acuerdo con el juicio de valor.

(3) [A y B salen juntos de escuchar una conferencia]

A: La conferencia ha sido estupenda

B: (silencio) 
En la interacción que se produce en las redes sociales, específicamente la que estamos analizando aquí, consideramos necesario señalar que las reacciones por medio de emoticonos constituyen intervenciones convocadas, pero no requeridas. Nos referimos con "convocadas" a que son intervenciones reactivas admitidas en el intercambio, posibilitadas por el sistema, condicionadas por el turno anterior (en términos de la pertinencia condicional) y, sobre todo, que el interlocutor - emisor del turno iniciativo - sabe que pueden recibirse pero que pueden también no aparecer. Lo que estamos aquí afirmando es que, por las características del medio, en la interacción en Facebook esta segunda parte del par adyacente no es esperada en el mismo grado o en la misma manera que en las interacciones cara a cara: su ausencia no implica las consecuencias en el intercambio y potencialmente en la relación entre los participantes que tendría la ausencia de respuesta verbal en (3). Diremos, por tanto, para dar cuenta de esta diferencia en el resto del artículo que los emoticonos son actos reactivos convocados, y no requeridos.

Este último rasgo de los emoticonos como intervenciones reactivas parece deberse, principalmente, a que en la interacción en Facebook el componente de toma de turno funciona de manera diferente a la prototípica en una conversación cara a cara: en la interacción en Facebook, lo más frecuente es que no haya selección de otro hablante para tomar el turno siguiente, sino que cualquiera de los participantes ratificados —en el caso de Facebook, cualquiera de los que tiene acceso al mensaje - puede autoseleccionarse, o puede que ninguno de ellos lo haga. ${ }^{6}$ Que no haya un interlocutor seleccionado para tomar el siguiente turno y haya, como suele ocurrir en estas interacciones, un amplio número de destinatarios potenciales que podrían autoseleccionarse - pero de los que ni siquiera se puede asegurar que hayan recibido el mensaje - hace que la expectativa de una reacción al turno iniciativo se relaje: no se requiere, en nuestros términos, el turno reactivo, sino que solo se convoca. Este punto será relevante para abordar, posteriormente, la interpretación asignada a la reacción me gusta durante el intercambio comunicativo.

6 No es imposible que haya selección de otro hablante, pero es una situación mucho menos frecuente y parece que, en ese caso, el valor de la ausencia de un turno reactivo sí se asemejaría más al de la conversación cara a cara. 


\section{El emoticono reactivo me gusta}

Este emoticono tiene en principio un significado convencionalmente definido, 'me gusta', que corresponde al icono de la mano con el dedo hacia arriba, asociado generalmente con una valoración positiva. La función con la que la red social hace accesible esta herramienta es la de reaccionar indicando agrado, por parte del usuario, ante contenidos expuestos por otros participantes.

\subsection{La valoración positiva y sus condiciones de adecuación}

Desde el punto de vista del funcionamiento de la interacción que hemos descrito brevemente en los apartados anteriores, la intervención reactiva cuyo único movimiento realiza el acto de expresar agrado sería esperada y admisible en una conversación bajo las siguientes dos condiciones:

La primera condición tiene que ver con la pertinencia condicional y, por tanto, con el tipo de intervención a la que se responde. Las valoraciones son respuestas pertinentemente condicionadas - y por tanto esperadas y admisiblesdespués de actos asertivos: descripciones, aserciones, narraciones, expresiones de opinión. Enunciados como los recogidos en (4) podrían, en un intercambio oral, recibir como respuesta una valoración positiva como ;Qué bien!:

(4) a. Estas son las mejores vacaciones de mi vida.

b. Gané el primer premio.

c. No va a llover mañana.

Del mismo modo, publicaciones en Facebook como las recogidas en los dos incisos de la Figura 4, equivalentes en términos ilocutivos a un acto asertivo, tienen como respuesta pertinentemente condicionada una valoración positiva.

En cambio, las valoraciones positivas no son respuestas pertinentemente condicionadas — esperadas o incluso aceptables, que completen el par adyacente - tras otros actos iniciativos como preguntas, las cuales esperan, en cambio, una respuesta como en (5a), ofrecimientos o invitaciones, que esperan aceptación o rechazo al ofrecimiento como en (5b), o actos directivos como peticiones $\mathrm{u}$ órdenes, que esperan un turno reactivo en el que se acceda o no a lo pedido 


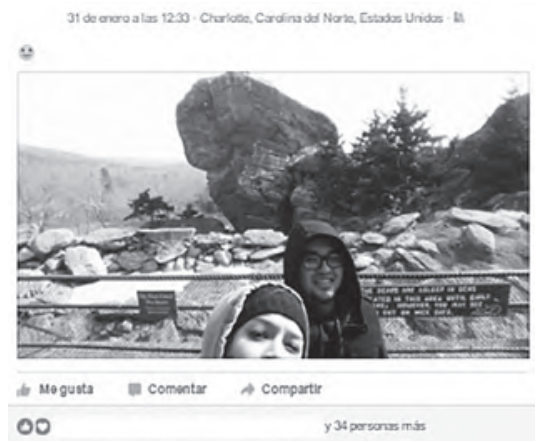

(a)

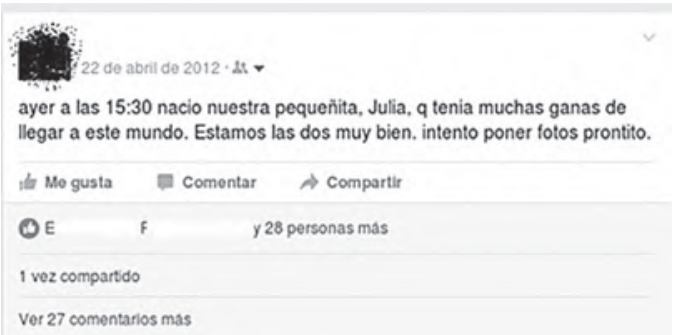

(b)

FIGURA 4. Publicaciones para las que la valoración positiva es una respuesta pertinentemente condicionada

como en $(5 \mathrm{c})$. En la conversación, resultaría extraño que se respondiera a las intervenciones de (5) con ;Qué bien!

(5) a. ¿Alguien tiene un lapicero?

b. ¿Quieres un café?

c. ¿Me acompañas al supermercado?

También en las publicaciones que constituyen actos iniciativos en Facebook aparecen actos como estos: los ejemplos de la Figura 5 ilustran actos con valor directivo: 5a ilustra una pregunta, $5 \mathrm{~b}$, un consejo o sugerencia y 5c, una petición. En ambos casos, según lo que acabamos de ver, la valoración positiva resultaría 


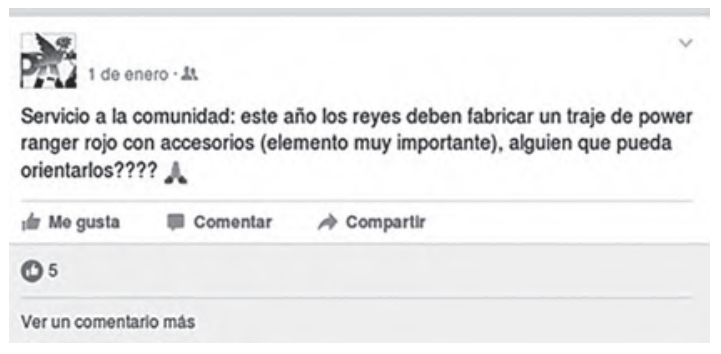

(a)

17:11 hrs reporta Maya
Hola buenas tardes
Percance en la carretera Naolinco - Xalapa en el órgano, una camioneta
volcada
PRECAUCIÓN
Me gusta $\quad$ Comentar
13

(b)

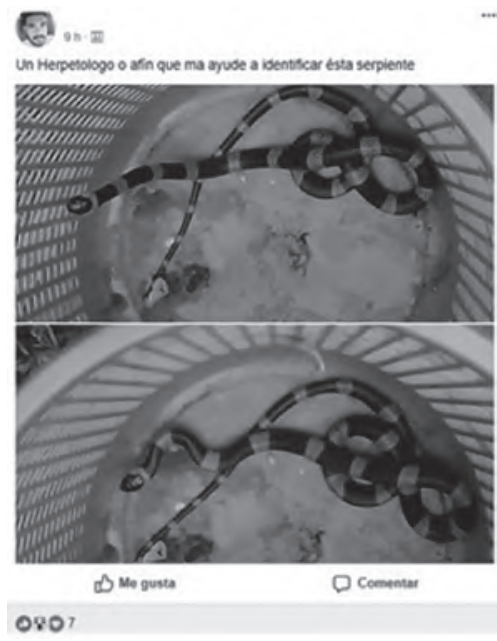

(c)

Figura 5. Publicaciones para las que la valoración positiva es una respuesta no pertinentemente condicionada 
una respuesta no pertinentemente condicionada y, por tanto, extraña, aunque en ambos vemos que la publicación ha recibido algunos me gusta como reacciones.

No en todos los casos es igualmente extraña la valoración como acto reactivo, ya que nuestra capacidad $-\mathrm{o}$ necesidad - de interpretar las intervenciones del interlocutor como relevantes hacen que, si la interpretación primera no es adecuada, busquemos otras posibles. Volveremos a este punto más adelante.

La segunda condición tiene que ver con el concepto de preferencia. Para que la reacción de expresar agrado sea aceptable debe haberse dado un turno iniciativo cuya respuesta preferida sea una valoración positiva. Esto dependerá no ya del tipo de acto, como en el punto anterior, sino del contenido de la intervención - y del conocimiento compartido de los hablantes. La expresión de agrado o valoración positiva — contenido convencional del emoticono reactivo me gusta - sería la respuesta preferida de turnos iniciativos en los que el contenido asertado (narrado, descrito, la opinión vertida) es positivo (6a, 6b), o en el caso de valoraciones o críticas de las que no es objeto el propio hablante (6c). En cambio, sería en principio una respuesta no preferida, que en el caso de aparecer verbalmente tendría una forma marcada, si el turno iniciativo narra, describe o comenta algo negativo para el hablante (Pomerantz, 1984). ${ }^{7}$ De nuevo, aceptaríamos como respuesta preferida una formulación directa como iQué bien! tras los enunciados de (6), pero no tras los de (7):

(6) a. Estas son las mejores vacaciones de mi vida

b. Gané el primer premio

c. Nuestro presidente es un auténtico desastre

(7) a. Estas son las peores vacaciones de mi vida

b. Perdí el concurso

c. Soy un auténtico desastre

7 Como se apuntó, se descarta en general en los trabajos previos la idea de que determinado tipo de intervención pueda ser catalogado, en términos generales, como preferido o no preferido, pero sí se describe el valor preferido de un tipo de intervención como respuesta a determinado tipo de acto. En este sentido, Pomerantz (1984) describe el valor preferido o no preferido de las valoraciones (positivas y negativas) según el tipo de intervención a la que respondan. 
El mismo contraste, ahora con datos de Facebook, se observa entre las Figuras $6 a, 6 b$ y $7 a, 7 b$.

Todo comenzó con una carrera loca que llamaban Spartan Race en el 2013. lo que llevó a convertir el Running en un estilo de vida a partir del 2014, el cual que llegó a un break en el 2016 y esperando volver pronto a recorrer más kilómetros!

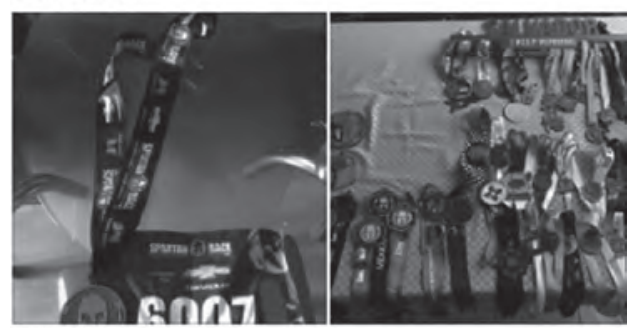

\begin{tabular}{|c|c|c|}
\hline in Me gusta & e comentar & $\rightarrow$ compartir \\
\hline $00 \%$ & & , 30 persores mis \\
\hline
\end{tabular}

(a)

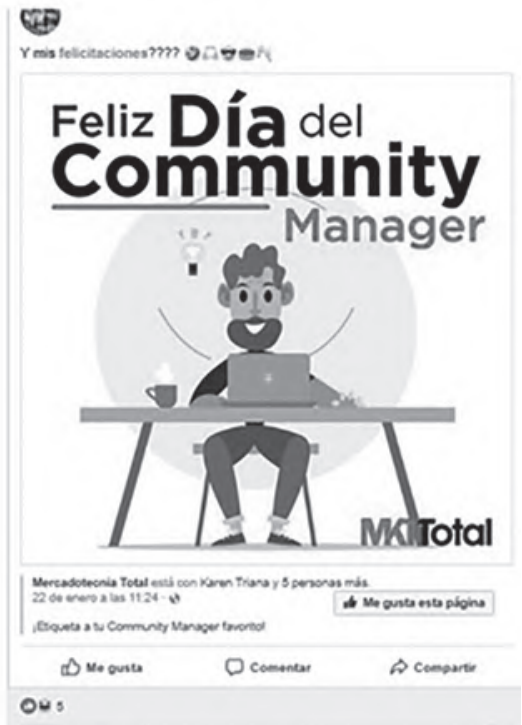

(b)

Figura 6. Publicaciones para las que la valoración positiva es una respuesta preferida
Con el permiso de la adminiatracion el di de hoy me rebarcon mi carro un tsuru color rojo brilante con placas YJB83333 se encontrabs estacionado en ta cale prolongacion av miguel alemin en to colonia ferrocarriera junto a to clinica 67 del IMSS y los taleres alstom si pueden compartir en otros grupos b agradeceria, dejen un puntto para que no se pierda la publicacion $s$
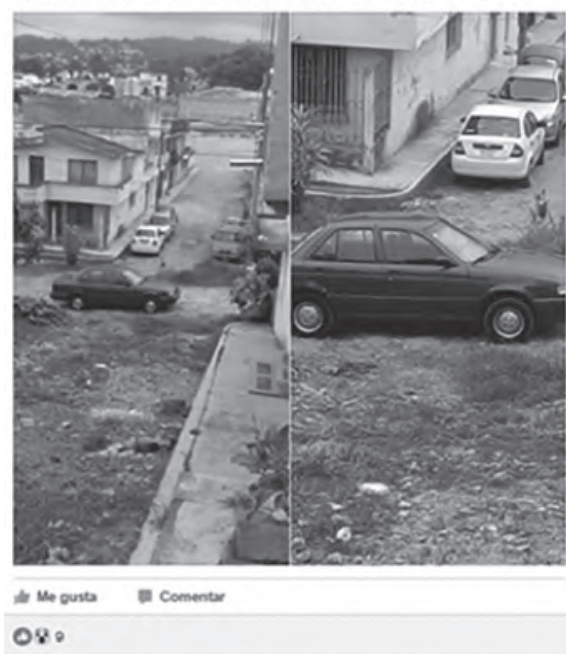

(a)

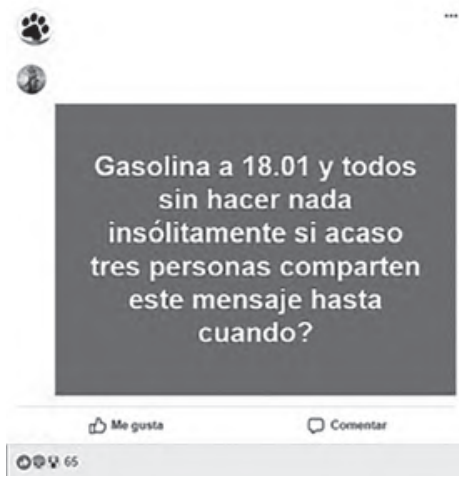

(b)

FIgURA 7. Publicaciones para las que la valoración positiva es una respuesta no preferida 
En resumen, si el movimiento realizado por medio del emoticono reactivo me gusta se interpreta con su significado literal, este movimiento solo resulta adecuado en las dos condiciones que acabamos de exponer. Por el contrario, me gusta no resultaría adecuado y, por tanto, no se encontraría o se encontraría con muy poca frecuencia si no se dan estas condiciones.

\subsection{El funcionamiento de me gusta más allá de su significado convencional}

Lo interesante del empleo del emoticono reactivo me gusta es que el comportamiento descrito en el apartado anterior no es el que, de hecho, se encontraba, y aún hoy se encuentra, en Facebook. Por el contrario, es frecuente que aparezcan pares adyacentes como los ilustrados en la Figura 5 o Figura 7, donde una pregunta y una mala noticia, respectivamente, reciben reacciones de me gusta.

El emoticono reactivo me gusta fue, y aún hoy es, empleado con frecuencia como a) intervención reactiva a turnos que no aceptarían una valoración para completar el par adyacente y b) respuesta a turnos para los que la valoración positiva constituiría una respuesta no preferida que, con el emoticono, se estaría realizando de forma directa, sin mitigación (esto es, sin las marcas más indirectas propias de la respuesta no preferida). ¿Cómo dar cuenta de estos empleos, frecuentes pero contrarios a lo esperado según el comportamiento interaccional habitual en los intercambios verbales descritos previamente?, ¿qué elementos del funcionamiento de la interacción entran en juego en la interpretación de estas intervenciones? Dado que el emoticono reactivo me gusta era la única opción para responder a otras intervenciones de forma rápida por medio de un clic, sin necesidad de escribir ni adjuntar archivos, los usuarios de Facebook pasaron a emplear el emoticono despojándolo de su significado semántico convencional. La intervención pasa a ser una reacción ambigua respecto a su contenido, cuya interpretación va a depender más de la posición que ocupa en la interacción y, específicamente, del contenido de turno anterior que del propio significado convencional del emoticono. En términos lingüísticos, podríamos decir que su interpretación va a depender más de un enriquecimiento pragmático — condicionado por el contexto de la interacción y ciertos principios sobre el funcionamiento de la misma interacción asumidos por los participantes- que por el contenido explícito y asociado literalmente a la forma del mensaje. 
Los principios que parecen guiar la aceptación de me gusta en usos como los ilustrados en la Figura 5 o en la Figura 7 son a) la tendencia a interpretar la respuesta del otro como pertinente y b) la asunción de que el emisor desea mantener buenas relaciones entre los interlocutores. Este segundo principio aplicará, o no, según las características de la interacción.

\subsubsection{Interpretar me gusta como pertinente: dos posibles interpretaciones no literales}

La tendencia a interpretar como pertinente un enunciado es un principio conversacional ampliamente compartido en pragmática y que se incluye en las diferentes propuestas de principios conversacionales que dan cuenta de la comunicación inferencial. ${ }^{8}$ En el caso que nos ocupa, la búsqueda de la pertinencia de la reacción me gusta, cuando la interpretación literal no resulta adecuada, puede satisfacerse de dos maneras.

Una primera estrategia interpretativa consiste en interpretar que la valoración no es hacia los contenidos, sino hacia el acto enunciativo: me gusta/valoro que realices ese acto. Un par de emisiones, como en el ejemplo (8), pueden ser juzgadas como coherentes al interpretar que B se alegra de que A le haga un ofrecimiento.

(8) A: ¿Quieres un café?

B: ¡Qué bien!

La misma interpretación es relativamente fácil de obtener con otros actos compromisivos — esto es, actos por los que el hablante se compromete a realizar una acción-, como puede observarse en el ejemplo (9).

(9) A: Te ayudo.

B: Qué bien.

8 Por ejemplo, en la categoría de relación y su máxima dentro del principio de cooperación de Grice (1975); en el principio de pertinencia o relevancia de Sperber y Wilson (1986); o en el concepto de coherencia pragmática presentado por Brown y Yule (1983). 
Con otro tipo de actos iniciativos, esta posibilidad va limitándose: puede recuperarse, aunque con más dificultad, con los actos directivos, como puede observarse en (10-A), y resulta más difícil con el tipo de preguntas que se ejemplifica en (10-A1).

(10) A: ¿Me acompañas al supermercado?

B: Qué bien. (interpretación: 'qué bien que me lo pidas')

A1: ¿Alguien tiene un lapicero?

B2: "? iQué bien!

La otra estrategia a la que los usuarios de me gusta recurren, en nuestra opinión y a la luz de su frecuente empleo en casos como el de la Figura 5a, es el vaciado semántico de me gusta, que pasa de significar una muestra de agrado o valoración positiva - del contenido o del acto- a emplearse exclusivamente como un retroalimentador o comentador (Gallardo, 1996: 26) para mostrar que se ha recibido el mensaje y procurar que la intervención del interlocutor no quede sin respuesta, pero sin intención de transmitir valoración positiva. El acto reactivo pasa casi solo a ser eso, un acto reactivo, sin contenido semántico y sobre el que, por tanto, no es posible evaluar si es adecuado o inadecuado, preferido o no preferido, sino que funciona como una marca de contacto: 'te he leído, sigo tu publicación'.

La motivación para poner en marcha las dos interpretaciones que acabamos de mencionar - a) me agrada el acto (no el contenido) y b) el mensaje ha sido recibido - puede ser, como hemos visto, salvaguardar, en términos de Grice (1975), una actitud cooperativa del interlocutor y así asignar una interpretación en la que la respuesta (el me gusta) sea pertinente, dado que al interpretarla literalmente no lo sería. Pero, además, estas mismas interpretaciones son también posibles y a ellas se recurre también —en nuestra opinión- en los casos en los que me gusta sí sería una respuesta pertinente —es decir, aquí, completaría el par adyacente - pero sería, como valoración positiva, una respuesta no preferida, como se ejemplifica en la Figura 7a tras la publicación que relata el robo del coche. Es, en estas ocasiones, cuando entra en juego de forma esencial el segundo principio mencionado arriba: "la asunción de que el emisor desea mantener buenas relaciones". 
5.2.2. Evitar la interpretación de me gusta como respuesta no preferida: la relación entre los participantes

En los estudios pragmáticos y de discurso, es central la idea de que la interacción tiene, además del objetivo de transmitir contenidos, la finalidad $-\mathrm{o}$ al menos el efecto- de construir o modificar las relaciones interpersonales. El deseo de mantener o establecer buenas relaciones con el interlocutor, o de evitar el conflicto, es uno de los pilares de las diferentes teorías de la cortesía lingüística que se han propuesto en las últimas décadas (Leech, 1983; Brown \& Levinson, 1987; Bravo, 2005).

Como apuntábamos en $\S 3$, el concepto de preferencia se ha relacionado en los trabajos previos con el efecto de un daño o amenaza a la imagen del interlocutor y, consecuentemente, el daño a la relación entre interactuantes. Heritage (1984), por ejemplo, sugiere que la preferencia está relacionada de forma clara con el mantenimiento o la amenaza de la imagen del interlocutor (Goffman, 1955). Del mismo modo, Brown y Levinson (1987: 38) sostienen que las acciones no preferidas son típicamente actos amenazantes de la imagen del interlocutor. La relación entre estos conceptos resulta clara: una acción amenazante de la imagen y una no amenazante de la imagen tienen efectos distintos en la relación entre los hablantes y en la continuidad de la interacción. Por eso, si una acción se percibe como amenazante de la imagen del interlocutor, es más probable que se realice con una forma marcada, o mitigada (forma de las acciones no preferidas), mientras que las acciones no amenazantes se realizarán con más frecuencia de manera directa y sin retraso (forma de las acciones preferidas).

En algunas de las interacciones en Facebook que analizamos, parece que la interpretación del turno reactivo me gusta está mediada por el hecho de que el destinatario piensa que el emisor del me gusta desea preservar una buena relación con él, esto se opone a situaciones en las que, por el contrario, no se asume tal intención - típicamente, porque esa relación no existía previamente. Veamos esto con más detalle.

En interacciones (especialmente en aquellas realizadas cara a cara) en las que un participante es explícitamente seleccionado para tomar el turno, este debe hacerlo y dar una respuesta pertinentemente condicionada, sea esta una respuesta preferida o una no preferida. Como ya se apuntó arriba, en la inte- 
racción en Facebook no hay, en general, selección del siguiente hablante por lo que, ante la disyuntiva de ofrecer una respuesta no preferida o un silencio (Levinson, 1983: 333), los usuarios pueden escoger con mayor libertad un silencio sin que eso tenga las repercusiones negativas que probablemente tendría una respuesta no preferida. Como se apuntó arriba, en esta interacción en la que hay una multiplicidad de interlocutores potenciales y ninguno de ellos está explícitamente seleccionado, la ausencia de respuesta no tiene el mismo efecto en la interacción que en las conversaciones cara a cara.

En las interacciones de Facebook en páginas personales, donde los usuarios ya han sido ratificados como amigos o conocidos en distintos grados por el propio usuario, lo esperado es que los interlocutores traten de mantener la buena relación que hizo que se aceptaran como "amigos" en la red social. En estas interacciones, en la interpretación de un me gusta a un turno iniciativo — para el que la expresión de agrado supondría una respuesta pertinente pero no preferida, por ejemplo, una mala noticia-, se recurre a alguna de las dos estrategias detalladas anteriormente: o la valoración positiva es hacia el acto (en este caso, por ejemplo, el acto de compartir esa noticia) o la intervención se ha emitido exclusivamente con la intención de hacer saber al usuario que se ha leído el mensaje. Supongamos, por ejemplo, una publicación como (11) en un muro personal que recibiera reacciones de me gusta.

(11) Hoy ha sido un día terrible: he chocado con el coche, me he roto el pie y al llegar a casa se había incendiado la cocina.

La suposición de que el "amigo de Facebook" que reaccionó a ese estatus no manifestaría públicamente alegría por la mala suerte del usuario — dado que eso supondría afectar negativamente la relación entre ambos- lleva al emisor de ese mensaje a interpretar el me gusta de alguna otra manera, en una interpretación que no sea la literal y, por tanto, en este par adyacente, la no preferida.

En el contexto de las interacciones en los muros privados, ofrecer una respuesta no preferida es costoso para sus participantes. En caso de hacerlo, se preferirá presumiblemente un comentario en el que el emisor pueda desplegar las estrategias mitigadoras que típicamente acompañan a este tipo de respuestas en la interacción. En este caso, el efecto del emoticono tiene una forma única y no 
puede mitigarse, por lo que un hablante cooperativo, en una interacción en Facebook, típicamente, prefiere omitir una respuesta que no sea requerida a dar una no preferida y directa. Con esto en mente, en los intercambios en sus muros privados con sus "amigos de Facebook", los usuarios bloquean la interpretación literal de me gusta cuando esta supondría una respuesta no preferida y, como en los casos en los que suponía una respuesta no relevante, interpretan esa reacción como un retroalimentador o como una valoración del acto de enunciación y no del contenido.

Nótese, en cambio, que en interacciones en perfiles públicos de Facebook (páginas de medios de comunicación, por ejemplo, o de empresas, etc.) la asunción de que el emisor desea mantener buenas relaciones no aplica, o no necesariamente aplica: en estos intercambios, resulta menos extraño que pueda obtenerse una interpretación de una reacción me gusta como una respuesta no preferida. Por ejemplo, noticias negativas por su contenido — como un deceso- pueden a veces recibir me gusta con intención de mostrar agrado hacia el contenido y, de este modo, se desatan polémicas de este tipo constantemente en redes sociales a partir de temas "sensibles".

Es difícil saber con qué frecuencia se recurre a cada una de estas estrategias -interpretar como retroalimentador o como valoración del acto de enunciación-, ya que puede darse el caso de que ambas se pongan en práctica a la vez o los usuarios no sean muy conscientes de a cuál de ellas responde su empleo de me gusta en determinadas ocasiones. Sin embargo, la alta frecuencia del uso del emoticono reactivo me gusta como reacción a todo tipo de actos iniciativos solo puede explicarse porque los usuarios recurren a estas interpretaciones y esos mecanismos son motivados, a su vez, por la asunción de un principio de relevancia y de un principio de "buenos deseos" que se mantienen, también, en las interacciones en Facebook.

\section{Los nuevos emoticonos: predicciones desde la teoría de la comunicación}

A pesar de que, como se ha explicado e ilustrado, el empleo de me gusta es común como reacción a turnos iniciativos para los que una valoración positiva no constituye una respuesta preferida, el vaciado semántico del emoticono no pareció haber llegado nunca a completarse, por lo que no era extraño encon- 
trar muestras de incomodidad ante estos empleos. Esta incomodidad entre los usuarios se reflejaba en las numerosas peticiones a Facebook, en internet, de un emoticono reactivo no me gusta, y como respuesta a esa demanda se ofrecen los nuevos emoticonos a los que nos referimos en \$2.1. El fragmento mostrado a continuación da muestra también de que la reacción me gusta se empleaba en estos contextos y de la incomodidad que, en ocasiones, este empleo generaba en los usuarios.

Now if you want to show support for your friend who lost her job, got a flat tire, punched a Subway employee and stubbed her toe all in the same hour, you won't look like a d--k for digitally saying "I like that all this terrible stuff happened to you!" You can just choose one of the new animated emojis and display actual human empathy. ${ }^{9}$

Lo que se ha expuesto en este artículo nos lleva a hacer dos comentarios finales respecto a la implementación de los nuevos emoticonos por parte de Facebook.

Ante las repetidas peticiones del emoticono reactivo no me gusta, Facebook ofreció la gama de reacciones que actualmente existe pero no incluyó en el paradigma el demandado no me gusta y justificó esta decisión diciendo que un botón con ese significado sería "muy negativo" e iría contra el espíritu de la red social. A la vez, Facebook reconoce que "not everything is likable" y justifica la ampliación de los emoticonos como una herramienta para mostrar empatía.

De nuevo, estas valoraciones se comprenden mejor aplicando los conocimientos sobre la interacción que se han expuesto en los apartados anteriores: de la respuesta de Facebook a los usuarios se desprende que la red social evitó el emoticono reactivo no me gusta esgrimiendo que, en términos del estudio de la interacción, los usuarios emplearían esta reacción para ofrecer respuestas no

9 "Ahora, si quieres mostrar apoyo a tu amiga que perdió el trabajo, a quien se le ha pinchado la rueda, que golpeó a un empleado de Subway y se golpeó el dedo todo en solo una hora, ya no parecerás un idiota por decir digitalmente "Me gusta que todas esas cosas terribles te hayan pasado'. Puedes elegir uno de los nuevos emojis animados y mostrar empatía humana" (la traducción es nuestra). Tomado de Jenna Mullins, "Why we'll never get a dislike button on Facebook", en E! News. Recuperado de https://www.eonline.com/news/742888/why-we-11never-get-a-dislike-button-on-facebook 
preferidas a los contenidos de otros usuarios. Las respuestas no preferidas, sin duda, resultan socialmente incómodas y dañinas y Facebook no deseaba contribuir a ese efecto.

Sin embargo, el reconocimiento de que no todo es susceptible de gustar se ajusta a la idea de que no me gusta podría emplearse también como respuesta preferida y es, de hecho, la respuesta preferida a muchos turnos iniciativos (actos asertivos de contenidos negativos, críticas, denuncias, etc.). Los comentarios de los usuarios y el comportamiento típico de los usuarios de Facebook en las interacciones, al menos en los muros privados, parecen indicar que la petición de un emoticono reactivo no me gusta estaba justificada por la necesidad de ofrecer respuestas preferidas, y no respuestas no preferidas y, por tanto, ofrecer respuestas que lejos de resultar dañinas para las relaciones entre usuarios permitieran mostrar acuerdo en muchas interacciones que de hecho se dan en la red social. La misma "empatía" que Facebook quiere facilitar con los nuevos emoticonos podría expresarse en muchos contextos con el emoticono reactivo de no me gusta.

Por último, comprender el papel de los emoticonos como intervenciones reactivas cuya interpretación está guiada, por un lado, por principios generales de la comunicación interaccional y, por otro, por las particularidades de la red social y los usos que los miembros de la sociedad hacen de ella, permite realizar ciertas predicciones respecto a la progresiva aceptación de los nuevos emoticonos ofrecidos por Facebook. La predicción es que el alto número de me gusta irá disminuyendo pero esto ocurrirá, en primer lugar, en aquellos contextos en los que, según su significado literal, la respuesta con me gusta es no preferida. Ante aquellos contenidos para los que la respuesta de una valoración positiva resulte inadecuada, los emoticonos que permitan ofrecer la respuesta preferida —me enoja, me entristece— previsiblemente irán volviéndose más frecuentes. En cambio, entre el emoticono reactivo me gusta y los emoticonos me divierte, me encanta, me sorprende, el empleo de me gusta se prolongará en el tiempo porque no resultan frecuentes los contextos en los que, con su significado literal, me gusta resulte una respuesta inadecuada y me divierte, me encanta o me sorprende una adecuada. 


\section{Conclusiones}

En este trabajo se ha analizado el empleo del emoticono reactivo me gusta en la red social Facebook aplicando los conocimientos sobre el funcionamiento de la interacción que se han desarrollado en el análisis conversacional y la pragmática. El análisis presentado en este trabajo se fundamenta en la idea general expuesta en los estudios de lengua en uso de que, en cada tipo de actividad (tomando el concepto de Levinson, 1979), sus propiedades condicionan las contribuciones que pueden hacerse a esa actividad y ayudan a determinar las inferencias que de esas contribuciones se obtienen, es decir, ayudan a establecer la interpretación de esas contribuciones.

El análisis del empleo de me gusta como intervención reactiva en las interacciones en Facebook revela diferentes interpretaciones, literal y no literales, de este emoticono y da cuenta, a su vez, de los elementos que condicionan - permiten o fuerzan - estas interpretaciones alternativas. Se ha expuesto que la interpretación literal como valoración positiva se obtiene cuando esta es una reacción pertinente y preferida, mientras que los mecanismos de búsqueda de pertinencia y la relación entre los usuarios (y el deseo - o no- de mantener una buena relación) dan cuenta de la reinterpretación de me gusta como intervención reactiva con características diferentes a las de la valoración positiva.

Abordar el empleo del emoticono reactivo desde la óptica del estudio de las interacciones contribuye a iluminar algunos aspectos del mismo que no se han considerado hasta el momento y permite hacer predicciones respecto a la adaptación de los nuevos emoticonos en la red social. Al mismo tiempo, analizar fenómenos comunicativos específicos de una interacción como la que se lleva a cabo en Facebook posibilita el avance hacia una caracterización de este tipo de actividad comunicativa, enormemente frecuente hoy y que, sin duda, presenta particularidades aún no convenientemente descritas. En definitiva, consideramos que este trabajo contribuye a la muy necesaria aplicación de la lingüística —en este caso, la pragmática - a la descripción del fenómeno tan presente hoy de la comunicación mediada por computadora. 


\section{Referencias}

Alturo, NúRIa (2010). Coherencia discursiva: dimensiones contextual, conceptual y gramatical. Círculo de Lingüística Aplicada a la Comunicación, 41, 3-30.

Atkinson, J. Maxell, \& Heritage, John (Eds.) (1984). Structures of social action: Studies in conversation analysis. Cambridge: Cambridge University Press.

Bravo, Diana (2005). Estudios de la (des)cortesía en español. Categorías conceptuales y aplicaciones a corpora orales y escritos. Buenos Aires: Dunken.

Brown, Gillian, \& Yule, George (1983). Discourse analysis. Cambridge: Cambridge University Press.

Brown, Penelope, \& Levinson, Stephen C. (1987). Politeness: Some universals in language usage. Cambridge: Cambridge University Press.

Coulthard, Malcolm (1977). An introduction to discourse analysis. Londres: Longman.

Church, Amelia (2004). Preference revisited. Rask, 21, 111-129.

Gallardo Paúls, Beatriz (1996). Análisis conversacional y pragmática del receptor. Valencia: Episteme.

Gallardo Paúls, Beatriz (1998). Comentario de textos conversacionales. I. De la teoría al comentario. Madrid: Arco/Libros.

Goffman, Erving (1955). On face-work: An analysis of ritual elements in social interaction. Psychiatry: Interpersonal and Biological Processes, 18(3), 213-231.

Goodwin, Charles, \& Heritage, John (1990). Conversation analysis. Annual Review of Anthropology, 19, 283-307.

Grice, Paul (1975). Logic and conversation. En Peter Cole \& Jerry L. Morgan (Eds.), Syntax and semantics: Speech acts (vol. 3, pp. 41-58). Nueva York: Academic Press.

Hengeveld, Kees \& Mackenzie, J. Lachlan (2008). Functional discourse grammar. A typologically-based theory of language structure. Oxford: Oxford University Press.

Heritage, John (1984). Garfinkel and Ethnomethodology. Cambridge: Polity Press.

Herring, Susan C. (2001). Computer-mediated discourse. En Deborah Tannen, Heidi E. Hamilton \& Deborah Schiffrin (Eds.), The handbook of discourse analysis (pp. 612-634). Oxford: Blackwell.

Herring, Susan C. (2007). A faceted classification scheme for computer-mediated discourse. Language@Internet, 4, artículo 1. Recuperado de http://www.languageatinternet.org/ articles/2007/761 
Hutchby, Ian, \& Wooffitt, Robin (1998). Conversation analysis: Principles, practices and applications. Cambridge: Wiley.

Kerbrat Orecchioni, Catherine (1990). Les interactions verbales. París: Armand Colin.

Kerbrat Orecchioni, Catherine (1996). La conversation. París: Seuil.

Kerbrat Orecchioni, Catherine (1998). La notion d'interaction en linguistique: origine, apports, bilan. Langue Française, 117, 51-67.

Leech, Geoffrey N. (1983). Principles of pragmatics. Londres: Longman.

Levinson, Stephen C. (1979). Activity types and language. Linguistics, 17(5-6), 365-369.

Levinson, Stephen C. (1983). Pragmatics. Cambridge: Cambridge University Press.

Novak, Petra Kralj; Smailović, Jasmina; Sluban, Borut, \& Mozetič, Igor (2015). Sentiment of emojis. PLOS ONE, 10(12), 1-22.

Pillet-Shore, Danielle (2017). Preference organization. En Jon Nussbaum (Ed.), The Oxford research encyclopedia of communication [en línea]. Nueva York: Oxford University Press. doi: 10.1093/acrefore/9780190228613.013.132

Pomerantz, Anita (1984). Agreeing and disagreeing with assessments: Some features of preferred/dispreferred turn shaped. En J. Maxwell Atkinson \& John Heritage (Eds.), Structures of social action: Studies in conversation analysis (pp. 57-101). Cambridge: Cambridge University Press.

SACKS, Harvey (1992). Lectures on conversation. Oxford: Blackwell.

Sacks, Harvey; Schegloff, Emanuel A., \& Jefferson, Gail (1974). A simplest systematics for the organization of turn-taking for conversation. Language, 50(4), 696-735.

Schegloff, Emanuel A. (1968). Sequencing in conversational openings. En John Gumperz \& Dell Hymes (Eds.), Directions in Sociolinguistics. The ethnography of communication (pp. 346-380). Hoboken: Wiley.

SPerber, Dan, \& Wilson, DeIrdre (1986). Relevance: Communication and cognition. Oxford: Blackwell.

Stivers, Tanya (2013). Sequence organization. En Jack Sidnell \& Tanya Stivers (Eds.), The handbook of conversation analysis (pp. 191-209). Hoboken: Wiley-Blackwell. 